\title{
Article \\ Effects of a Single Session of Floss Band Intervention on Flexibility of Thigh, Knee Joint Proprioception, Muscle Force Output, and Dynamic Balance in Young Adults
}

\author{
Nai-Jen Chang ${ }^{1,2,3,4, *} \mathbb{D}$, Wei-Chun Hung ${ }^{1}$, Chia-Lun Lee ${ }^{5}\left(\mathbb{D}\right.$, Wen-Dien Chang ${ }^{6} \mathbb{D}$ and Bo-Han Wu ${ }^{7}$ \\ 1 Department of Sports Medicine, Kaohsiung Medical University, Kaohsiung 807, Taiwan; \\ a254456k2@gmail.com \\ 2 Ph.D. Program in Biomedical Engineering, College of Medicine, Kaohsiung Medical University, \\ Kaohsiung 807, Taiwan \\ 3 Regenerative Medicine and Cell Therapy Research Center, Kaohsiung Medical University, \\ Kaohsiung 807, Taiwan \\ 4 Department of Medical Research, Kaohsiung Medical University Hospital, Kaohsiung 807, Taiwan \\ 5 Center for Physical and Health Education, National Sun Yat-sen University, Kaohsiung 804, Taiwan; \\ karenlee1129@gmail.com \\ 6 Department of Sport Performance, National Taiwan University of Sport, Taichung 404, Taiwan; \\ changwendien@gmail.com \\ 7 Department of Recreational Sport \& Health Promotion, National Pingtung University of Science \\ and Technology, Pingtung 912, Taiwan; licar@mail.npust.edu.tw \\ * Correspondence: chang.naijen@gmail.com; Tel.: +886-7-312-1101 (ext. 2646); Fax: +886-7-313-8359
}

Citation: Chang, N.-J.; Hung, W.-C.; Lee, C.-L.; Chang, W.-D.; Wu, B.-H. Effects of a Single Session of Floss Band Intervention on Flexibility of Thigh, Knee Joint Proprioception, Muscle Force Output, and Dynamic Balance in Young Adults. Appl. Sci. 2021, 11, 12052. https://doi.org/ 10.3390/app112412052

Academic Editor: Redha Taiar

Received: 10 November 2021 Accepted: 13 December 2021 Published: 17 December 2021

Publisher's Note: MDPI stays neutral with regard to jurisdictional claims in published maps and institutional affiliations.

Copyright: () 2021 by the authors. Licensee MDPI, Basel, Switzerland. This article is an open access article distributed under the terms and conditions of the Creative Commons Attribution (CC BY) license (https:// creativecommons.org/licenses/by/ $4.0 /)$.

\begin{abstract}
The floss band (FB) has been correlated with increases in the joint range of motion (ROM). However, the literature on FB effectiveness in knee joint ROM and athletic performance remains sparse. This study investigated the effects of FB on the flexibility of the quadriceps and hamstrings, knee joint proprioception, muscle force output, and dynamic balance in men. Thirty recreationally active men without musculoskeletal disorders were randomized to receive FB (Lime Green; Sanctband flossband) and elastic bandage (EB) intervention on the dominant knee joint. Participants received two interventions on two occasions with 2 days of rest between interventions. The primary outcome was the flexibility of the quadriceps and hamstrings; the secondary outcomes were knee proprioception (joint reposition angle error), knee muscle force output, and dynamic balance. Preintervention and postintervention (immediately following band removal and $20 \mathrm{~min}$ later) measurements were obtained. After FB intervention, hamstring flexibility (immediately: $p<0.001 ; 20$ min later: $p<0.001$ ) and quadriceps flexibility (immediately: $p<0.001 ; 20$ min later: $p<0.001$ ), quadriceps muscle force output (immediately: $p=0.007 ; 20$ min later: $p<0.001$ ), and dynamic balance (both immediately and 20 min later, $p<0.001$ ) were significantly improved. Compared with EB intervention, FB intervention significantly improved knee extension ROM (immediately and 20 min later, both $p<0.001$ ), knee flexion ROM (immediately, $p=0.01 ; 20$ min later, $p=0.03$ ), hamstrings muscle force output (20 $\mathrm{min}$ later, $p=0.022$ ) and dynamic balance (immediately, $p=0.016 ; 20 \mathrm{~min}$ later, $p=0.004$ ). Regarding proprioception, no significant difference among time points and conditions was observed. In conclusion, FB intervention can significantly improve the flexibility of the quadriceps and hamstrings, quadriceps muscle force output, and dynamic balance without impeding knee proprioception. Physiotherapists or athletic professionals may consider FB intervention as a potential tool as a warmup to enhance the flexibility of the quadriceps and hamstrings, quadriceps muscle force output, and dynamic balance in young adults.
\end{abstract}

Keywords: sports rehabilitation; warm-up exercises; tissue flossing; blood flow restriction; health promotion 


\section{Introduction}

Sound muscle flexibility is a critical issue in athletic settings, and it determines the athletic performance of the lower-limb biomechanics [1]. In recent years, the floss band (FB) has been popularized by authors Starrett and Cordoza, who claim that the FB can increase range of motion (ROM), strength, and jumping performance and can enhance fatigue recovery [2,3]. The FB can be applied on a joint and on soft tissue. The FB technique consists of tightly wrapping part of a limb or a joint with a thick elastic band, resulting in fractional vascular occlusion of the blood flow distal to the wrapped area [4]. The reperfusion effect, fascial shearing, and occlusion of blood to the muscle may be the physiological mechanisms that underlie flossing [5]. When FB intervention involves passive twisting with the FB and active functional movements with a joint or muscle tightly wrapped by the FB for approximately $2-5 \mathrm{~min}$, this technique could produce a localized effect of vascular occlusion and blood flow restriction and affect the relationship of the fascia with the neuromusculoskeletal system; this effect allows the fascia to stretch and move freely [5]. When the FB is loosened and the pressure is released, blood quickly flows back to the oppressed site, and this return of blood flow may increase localized microenvironmental changes, such as growth hormone levels and the catecholamine response; this blood flow may also increase muscle strength and muscle contraction efficiency [6-9]. Moreover, studies have indicated that this process may increase the ROM of the joints. Therefore, pressure may be employed with the FB to compress the soft tissue to achieve the ischemic effect on the tissue [10,11]; subsequently, actions and stretches must be used to treat the localized part, remove the adhesion, allow the myofascial connective tissue to slide, and then restore the original muscle elasticity [11].

Despite a lack of empirical evidence, tissue flossing is widely performed among athletes because of its immediate benefits. According to a scoping review, floss band use likely can enhance joint ROM but with a small to moderate effect on ankle, calf, thigh, and shoulder ROM [12,13]. The FB may be similar to blood flow restriction (BFR), but it is known to be used more frequently in warmups for promising outcomes [14]. Diller et al. discovered that an FB could increase the ankle dorsiflexion angle by $6 \%$, weight-bearing lunge value by $14 \%$, and single-leg jump velocity by $6 \%$ in recreational athletes, which are all significant changes [15]. Vogrin et al. indicated that an FB applied to the ankle is an effective method to increase ankle ROM [16]. Kaneda et al. demonstrated that an FB used on hamstring muscles is more beneficial than dynamic stretching with respect to increasing hamstring muscle flexibility in healthy young men [5]. By contrast, another crossover study by Mills indicated limited support for the use of an FB to improve ankle $\mathrm{ROM}$, countermovement jumping, and sprinting performance up to $30 \mathrm{~min}$ postapplication in elite rugby union athletes.

The effectiveness of flossing remains inconclusive. A recent systematic review and meta-analysis published by Kielur and Powden focused on changes of ankle dorsiflexion using FB, but they did not investigate other outcome variables such as balance, muscle force output, or functional tests [17]. Taken together, substantial research on FB has been emerging, but the literature on FB effectiveness in knee joint ROM and athletic performance has yet to be evaluated, particularly in the knee joint in adults. To understand the effectiveness behind the $\mathrm{FB}$, the objectives of this study were to investigate the effects of an FB on the flexibility of the quadriceps and hamstrings, knee proprioception, dynamic balance, and maximal muscle force output in recreationally active men.

\section{Materials and Methods}

\subsection{Participants}

The study protocol was approved by the Kaohsiung Medical University Hospital Institutional Review Board (KMUHIRB-F(I)-20190063) and was conducted in accordance with the principles of the Declaration of Helsinki. Thirty recreationally active men (age $24.1 \pm 1.7$ years old; body height $174.2 \pm 0.1 \mathrm{~cm}$; body mass $77.3 \pm 16.0 \mathrm{~kg}$; BMI $25.4 \pm 4.5$ ) were recruited to participate. Participants were recruited through verbal communication 
and posters placed around a university campus. "Recreational" was defined as performing regular physical exercise sessions at least 3 times weekly [15]. Participants who had had no serious lower-extremity musculoskeletal system problems, neurological problems, cardiopulmonary disease, open wounds, acute injuries, history of tumors, pain due to metastasis or radiation, or use of therapeutic drugs (i.e., non-steroidal anti-inflammatory drugs) 6 months prior to the study were included. Before the assessment, the examiner explained the purpose, content, and procedure of the assessment in detail to the participants, and then participants completed the consent form regarding the benefits and risks of this study before proceeding with the assessment.

\subsection{Study Procedures}

This study employed a crossover design. Participants randomly performed two distinct interventions using an FB and an elastic bandage (EB). The main differences between the two wraps are the material texture and the tension applied in tissue flossing (blood flow restriction). FBs are made of natural rubber or latex and are available in various lengths, widths, and thicknesses [18]. EB is made of a cotton and elastic yarn mix [19]. EB must be applied using gentle, comfortable wrapping because excessive tightness impedes limb circulation. Clinically, EB is used for edema control, wound dressings, and muscle and joint support. Therefore, the wrapping duration tends to be longer than that of FBs. FB is used in myofascial compression interventions and rehabilitation and by sports medicine professionals, where they are used in tissue flossing interventions (lasting 2-5 min) that may also include functional movements of the wrapped limb. For effective tissue flossing, FB exhibits greater tension when stretched (stretch force range of $50 \%$ to $90 \%$ of the band length) relative to EB $[13,18,20]$. Therefore, precautions should be taken during FB use. Prior to the start of the assessment session, participants underwent a familiarization session in which they were instructed on how to use an FB and an EB. During this orientation, participants were familiarized with the procedures and practiced using the assessment tools and equipment of the study. One day after the familiarization session, each participant completed two assessment sessions in a randomized order, with $48 \mathrm{~h}$ of rest between each session. Participants threw a die to the randomized exercise assignment by themselves. Odd numbers represented EB; even numbers represented FB [21].

Participants were requested to avoid vigorous activities for $24 \mathrm{~h}$ before each assessment session. Before beginning the experiment, each participant was instructed to perform five sets of knee flexion and extension at an angular velocity of $180^{\circ} / \mathrm{s}$ on a Biodex system (Mirion Technologies, Shirley, NY, USA) as a general warmup. Subsequently, participants performed the preintervention measurements in the following order: knee joint ROM, proprioception, isokinetic muscle strength test, and Y-balance test (YBT). After completion of the preintervention measurements, participants completed the intervention (FB or EB) in a randomized order. Postintervention measurements were conducted in the same order as preintervention measurements: immediately after the intervention and 20 min later.

\subsection{Outcome Measures}

Before initiating data collection, the researchers received comprehensive training on conducting the flexibility of the quadriceps and hamstrings, knee proprioception, and isokinetic muscle strength tests as well as the YBT. A clinical physiotherapist was also trained and certified by means of the Easy Flossing Instructor Course.

\subsubsection{Primary Outcome}

The primary outcome was flexibility of the quadriceps and hamstrings. The hamstring flexibility was measured using the popliteus test, which considers the flexibility of the hamstrings. This test has a high intraclass correlation coefficient (ICC; 0.90) [22]. Each participant assumed a lying position, and the dominant hip and knee joints were flexed at $90^{\circ}$. Subsequently, an examiner placed the reference point of the universal goniometer on the lateral epicondyle of the femur, with the fixed end facing the medial line of the thigh, 
and the moving end aligned with the fibula midline lateral malleolus. Each participant actively straightened the investigated leg upward until they felt some discomfort but no pain. The angle between the thigh and leg was measured twice and calculated to obtain an average. For the quadriceps flexibility, Ely's test was used for the flexibility of the quadriceps. This test has a high ICC (0.91) [23]. In this test, each participant was asked to lie down and place the dominant leg in a straight position. The center point of the goniometer was aligned with the lateral epicondyle of the femur; the fixation was aligned with the greater trochanter; and the moving arm was aligned with the lateral malleolus. Each participant actively bent the knee joint as far as possible to reach desired tightness but not too tight as to reach an uncomfortable range without any compensations (e.g., hip abduction, hyper-lordosis of the lumbar spine, or pelvic anterior tilt) [24].

\subsubsection{Secondary Outcomes}

The secondary outcomes were proprioception, muscle force output, and dynamic balance. For the proprioception test, the Biodex isokinetic dynamometer (Biodex System, Shirley, NY, USA) was used to assess knee active joint proprioception. Each participant sat in an upright position, and the dominant leg was on the test arm of the Biodex dynamometer. The thigh was fixed with a strap, and the calf was fixed to the movable arm to reduce compensatory body movements. Participants were asked to wear an eye mask to isolate their visual input. Subsequently, each participant actively moved the limb to the target angle of $45^{\circ}$ of flexion and then returned to $90^{\circ}$ of knee flexion [25]. The test was assessed three times and the results averaged. The joint reposition angle error, which is the difference between the setting and the perceived angle, was recorded as an index of knee proprioception [26].

The Biodex isokinetic dynamometer was also used in the knee isokinetic muscle strength test. The isokinetic quadriceps and hamstring muscles were measured at $60^{\circ} / \mathrm{s}$ using the Biodex system, which has high ICC values. [24]. The posture setting was the same as that for the proprioception test, but participants removed the eye mask. The dominant leg was tested. The participants were asked to actively perform knee extension and immediate knee flexion as rapidly as they could three times at a set angular velocity of $60^{\circ} / \mathrm{s}$. The highest peak torque value was recorded by means of the Biodex software, used in the statistical analysis, and further normalized by participants' body weight [27].

To assess the dynamic balance of the lower limbs, the YBT had a high ICC of 0.91 [23]. In this test, participants were required to maintain single-leg balance in their dominant leg. They were asked to be barefoot to eliminate the influence of shoes on the stability and balance of the lower limbs. First, participants stood on the central standing board and maintained balance, and then they moved the nondominant foot (moving foot) as far as possible from the origin to the front side. After returning the foot to the center point, participants extended in three directions (i.e., anterior, posterolateral, and posteromedial) (Figure 1). Six trial practices of the YBT were allowed for their dominant leg in the familiarization session to eliminate the learning effect [28]. When the participants performed the test, they let their toes slide with ease, extend to the farthest point, and return to the original position while maintaining balance. The measurement for each direction was obtained three times, and the data were averaged. The YBT composite score was calculated by averaging the three distances reached and normalizing the outcomes according to the lower limb length (measured from the anterior superior iliac spine to the medial malleolus in the supine position) and multiplied by 100 . 


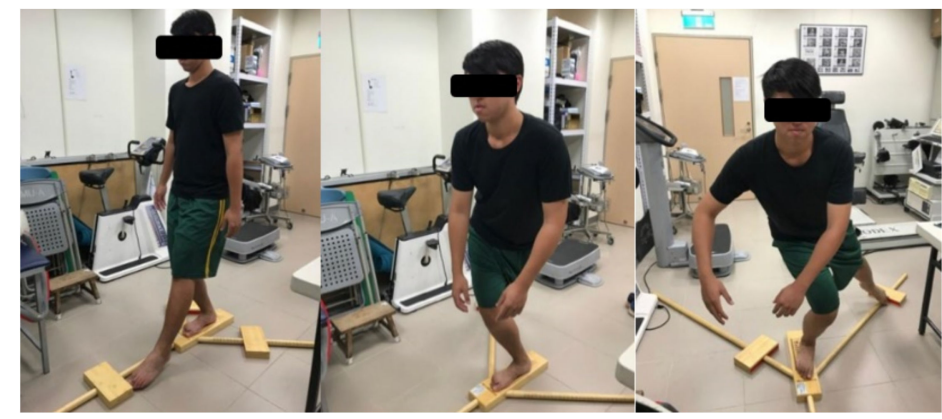

Figure 1. Y-Balance Test (YBT) in three directions (i.e., anterior, posterolateral, and posteromedial).

\subsection{Intervention Protocols}

\subsubsection{Elastic Bandage Intervention}

Participants used a 4-inch EB as the control condition on the dominant leg (Figure 2) and used a stretch that allows for conformity and comfort according to manufacturer instructions. The EB is a made of mixed with cotton and elastic yarn [19]. The band tension overlapped half of the previous part of the band, distally to proximally. Participants performed three functional movements: walking knee lift, side squat, and lunge [15]. All movements were monitored to ensure 10 repetitions in $3 \mathrm{~min}$ at 30 beats per $\mathrm{min}$, as measured using a metronome.

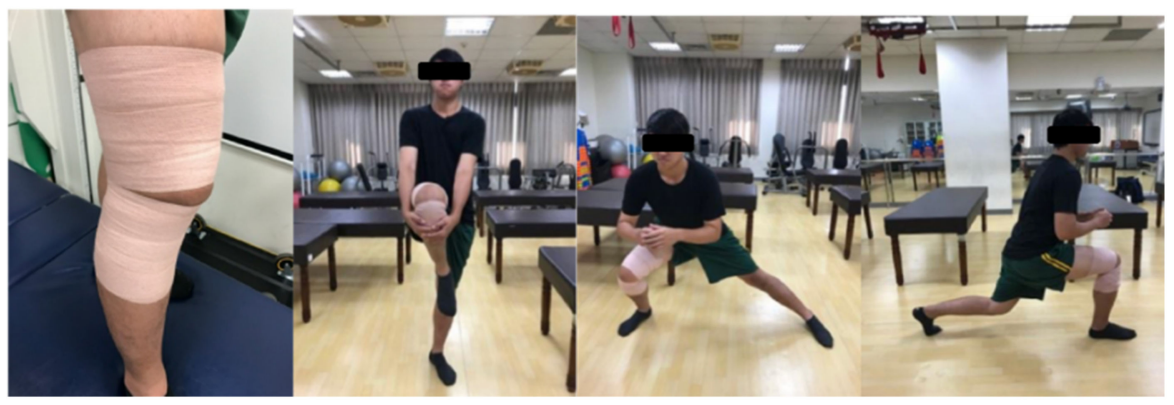

Figure 2. Elastic bandage intervention as the control condition.

\subsubsection{Floss Band Intervention}

In this study, the FB (Lime Green; Sanctband flossband) is made of natural rubber or latex $[18,20]$ and was adopted on the dominant leg as the same place as EB condition. A 2-inch Sanctband Malaysia Floss Band (Sanctband, Perak, Malaysia) was wrapped on participants' dominant leg (Figure 3). Assessment examiners followed the method suggested by Sanctband; they started from the tibial tuberosity on the dominant side, bound the band from the distal end to the proximal direction, leaving the patella uncovered, and continued to wrap to $5 \mathrm{~cm}$ above the lateral epicondyle of the femur. We used the Smart Handle Pro ZSH-ZP (Delta Industries, King of Prussia, PA, USA) to control the tension of the stretched FB. The stretch force was approximately $75 \%$ elongated and overlapped half of the previous part of the band, distally to proximally. The average band tension was approximately $40 \mathrm{~N}$ [29], and participants performed three functional movements: walking knee lift, side squat, and lunge [30]. All movements were monitored to ensure 10 repetitions in $3 \mathrm{~min}$ at 30 beats per min, as measured using a metronome, operating under the condition that if participants felt discomfort, sensory disturbances, whitening, or intense stabbing pain during wrapping, the FB would be removed immediately $[13,20]$. 

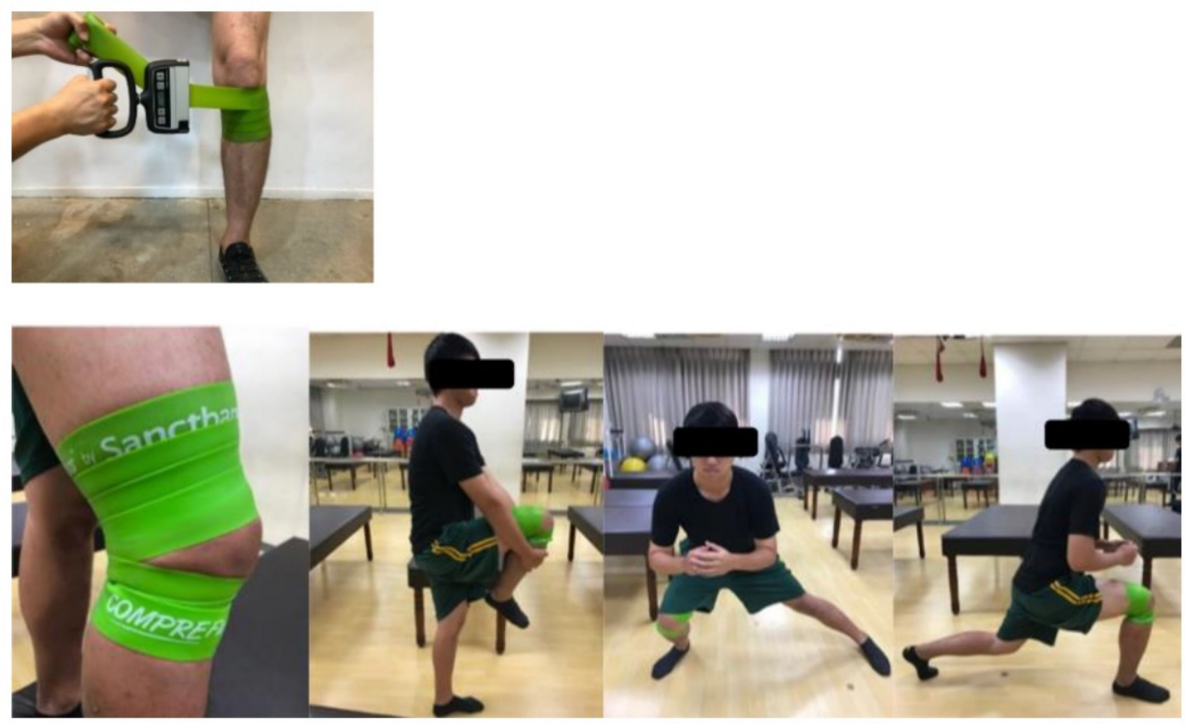

Figure 3. Sanctband floss band intervention.

\subsection{Statistical Analyses}

A priori sample size calculation was based on the anticipated differences in knee extension, with an anticipated large effect (effect size $=0.8$ ) based on our pilot study on the primary outcome between preintervention and postintervention measurement. An alpha level of 0.05 with two-tailed test and a desired statistical power of $90 \%$ were used in $G^{*}$ Power software to calculate the ideal sample size [31]. The minimum sample size was 19 participants. Assuming a dropout rate of 10\% [32], we enrolled 30 participants to increase statistical strength.

Statistical analyses were performed using SPSS software version 21.0 (IBM, Chicago, IL, USA). Data were assessed for normality (Shapiro-Wilk's test, $p>0.05$ ), and homogeneity of variance was confirmed using the Levene test. All data are presented as mean \pm standard deviation unless indicated otherwise. If the results of the Mauchly's sphericity test indicated that the sphericity assumption was violated, the Greenhouse-Geisser adjustment was used to correct the degrees of freedom. A (condition factor: FB vs. EB) $\times 3$ (time factor: preintervention vs. postintervention immediately vs. postintervention 20 min later) two-way repeated measure analysis of variance was adopted to assess the effects of various conditions on dependent variables. Moreover, the Bonferroni correction was used for post hoc testing to control type I error because multiple comparison methods were employed [33]. We calculated the amount of change from the baseline as $\frac{\text { (postintervention- preintervention) } \times 100 \%}{\text { preintervention }}$. The estimates of effect size (ES) used Cohen's $d(d=\mathrm{M} 1-\mathrm{M} 2 /$ opooled) to represent the magnitude of the effect and interpreted the ES as small $(d=0.2)$, medium $(d=0.5)$, and large $(d=0.8)$ [34]. The significance level of all data was set at $\alpha<0.5$.

\section{Results}

A flowchart of the experimental design is exhibited in Figure 4. The results of all outcomes are presented in Tables 1 and 2. The baseline of participants was comparable in all outcomes. All participants completed the study and none reported adverse events. No patient discomfort, sensory disturbances, whitening, intense stabbing pain, or skin allergy were reported during or after FB or EB intervention. 


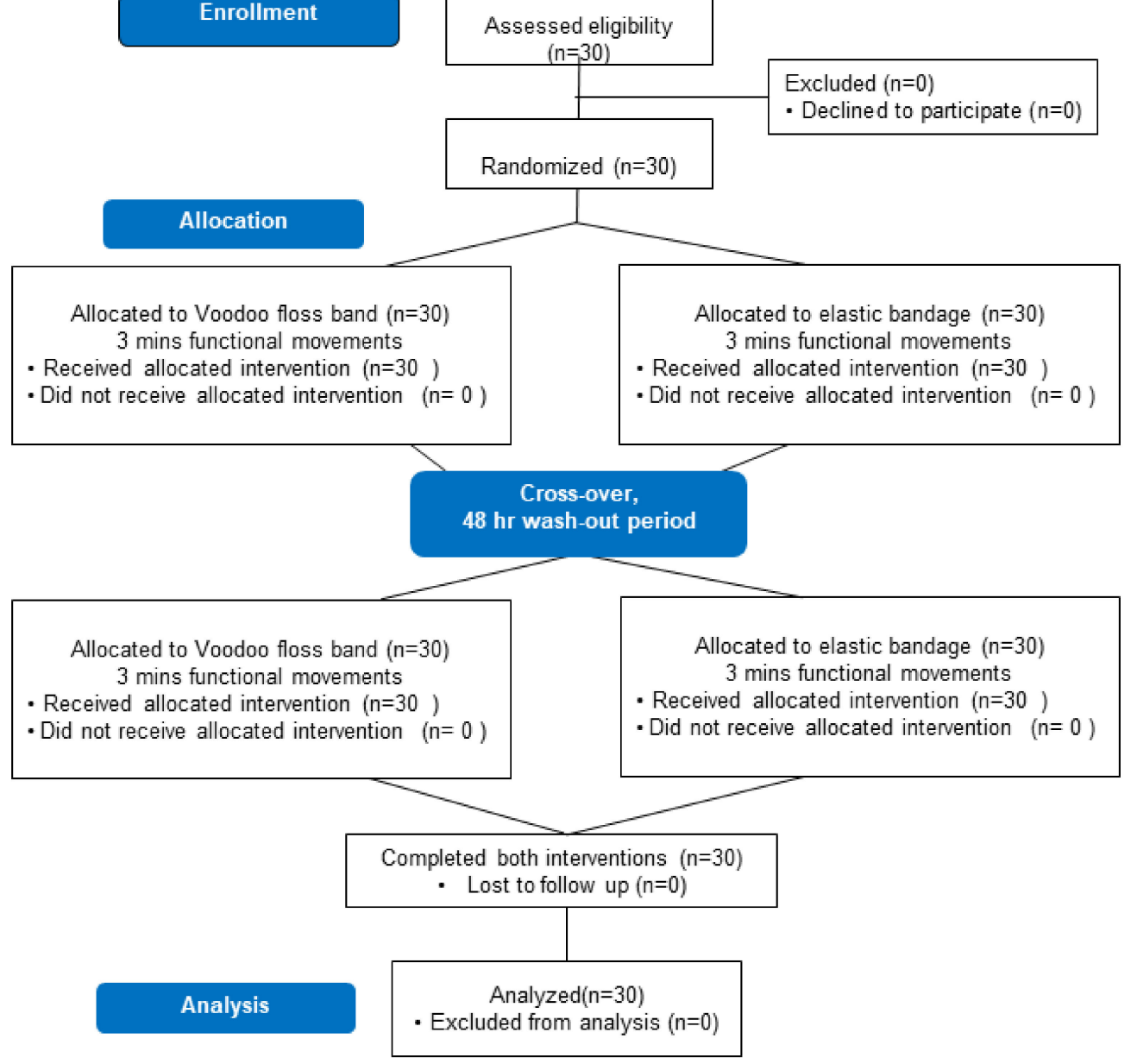

Figure 4. Flowchart of experimental design.

Table 1. Preintervention and postintervention measurements descriptive results.

\begin{tabular}{|c|c|c|c|c|c|c|c|c|c|}
\hline & ameter & Intervention & Pre & $\begin{array}{c}\text { Post } \\
\text { Immediately }\end{array}$ & $\begin{array}{c}\text { Post } \\
20 \mathrm{~min}\end{array}$ & $\stackrel{p}{\text { Immediately }}$ & $\begin{array}{c}p \\
\text { Post } \\
20 \mathrm{~min}\end{array}$ & $\begin{array}{c}\text { Effect Size } \\
\text { Immediately }\end{array}$ & $\begin{array}{l}\text { Effect } \\
\text { Size } \\
20 \mathrm{~min}\end{array}$ \\
\hline \multirow{4}{*}{ 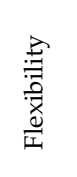 } & \multirow{2}{*}{$\begin{array}{c}\text { Hamstring } \\
\text { (degrees) }\end{array}$} & FB & $147 \pm 10.69$ & $155.06 \pm 9.36$ *\# & $154.7 \pm 10.8 * \#$ & $<0.001$ & $<0.001$ & 0.8 & 0.72 \\
\hline & & EB & $149.06 \pm 12.58$ & $147.06 \pm 11.17$ & $147.58 \pm 11.03$ & 0.079 & 0.689 & 0.17 & 0.13 \\
\hline & \multirow{2}{*}{$\begin{array}{l}\text { Quadriceps } \\
\text { (degrees) }\end{array}$} & $\mathrm{FB}$ & $129.81 \pm 10.69$ & $132.67 \pm 6.74$ *\# & $132.77 \pm 6.92$ *\# & $<0.001$ & $<0.001$ & 0.32 & 0.33 \\
\hline & & EB & $129.4 \pm 7.9$ & $129.39 \pm 8.19$ & $129.52 \pm 8.6$ & 1 & 1 & 0 & 0.01 \\
\hline \multirow{2}{*}{ 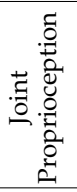 } & \multirow{2}{*}{$\begin{array}{l}\text { Joint } \\
\text { reposition } \\
\text { error } \\
\text { (degrees) }\end{array}$} & FB & $4.42 \pm 2.17$ & $3.88 \pm 2.13$ & $4.97 \pm 4.19$ & 0.39 & 0.76 & 0.25 & 0.16 \\
\hline & & EB & $3.69 \pm 2.34$ & $4.29 \pm 2.61$ & $4.25 \pm 2.31$ & 0.47 & 0.42 & 0.24 & 0.24 \\
\hline \multirow{4}{*}{ 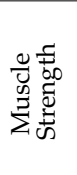 } & \multirow{2}{*}{$\begin{array}{l}\text { Quadriceps } \\
\text { (N-m/Kg) }\end{array}$} & FB & $1.22 \pm 0.43$ & $2.19 \pm 0.56^{*}$ & $1.38 \pm 0.47^{*}$ & 0.007 & $<0.001$ & 1.94 & 0.36 \\
\hline & & EB & $1.25 \pm 0.37$ & $2.13 \pm 0.58 *$ & $1.35 \pm 0.43^{*}$ & 0.007 & $<0.001$ & 1.8 & 0.25 \\
\hline & \multirow{2}{*}{$\begin{array}{l}\text { Hamstrings } \\
(\mathrm{N}-\mathrm{m} / \mathrm{Kg})\end{array}$} & $\mathrm{FB}$ & $2.12 \pm 0.52$ & $1.37 \pm 0.31 *$ & $2.12 \pm 0.46^{\#}$ & $<0.001$ & 1 & 1.75 & 0 \\
\hline & & EB & $2.12 \pm 0.49$ & $1.34 \pm 0.34$ * & $1.95 \pm 0.56$ * & $<0.001$ & $<0.001$ & 1.83 & 0.32 \\
\hline \multirow{2}{*}{ 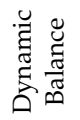 } & \multirow{2}{*}{$\begin{array}{l}\text { Y-balance } \\
\text { test }\end{array}$} & FB & $0.93 \pm 0.07$ & $0.97 \pm 0.07$ *\# & $0.99 \pm 0.07$ *\# & $<0.001$ & $<0.001$ & 0.57 & 0.86 \\
\hline & & EB & $0.94 \pm 0.07$ & $0.95 \pm 0.07$ * & $0.96 \pm 0.08$ & 0.25 & 0.208 & 0.14 & 0.27 \\
\hline
\end{tabular}

Data reported as mean \pm SD. ${ }^{*}$ Significant difference $(p<0.05)$ compared with preintervention. \# Significant difference $(p<0.05)$ compared with EB $(p<0.05)$ at same time point. Effect size: $d=\mathrm{M} 1-\mathrm{M} 2 / \sigma_{\text {pooled. }}$ Hamstring flexibility was measured using the popliteus test, which measures popliteal angle (degree) between the thigh and leg. Quadriceps flexibility was measured using Ely's test, which measures the knee flexion angle (degree) between the thigh and leg. 
Table 2. Comparison of postintervention with preintervention values.

\begin{tabular}{|c|c|c|}
\hline & $\begin{array}{c}\text { Post Immediately } \\
\Delta \text { FB- } \Delta \text { EB } \\
\text { (Effect Size) }\end{array}$ & $\begin{array}{c}20 \text { min Later } \\
\Delta \text { FB- } \Delta \text { EB } \\
\text { (Effect Size) }\end{array}$ \\
\hline Hamstring flexibility (degree) & $\begin{array}{l}10.06 \pm 5.3 \\
(2.09)\end{array}$ & $\begin{array}{l}9.18 \pm 8.81 \\
(1.58)\end{array}$ \\
\hline Quadriceps flexibility (degree) & $\begin{array}{l}2.87 \pm 5.31 \\
\quad(0.76)\end{array}$ & $\begin{array}{l}2.83 \pm 4.22 \\
(0.84)\end{array}$ \\
\hline Joint proprioception (degree) & $\begin{array}{c}-0.96 \pm 4.06 \\
(-0.24)\end{array}$ & $\begin{array}{l}0.17 \pm 5.52 \\
\quad(0.04)\end{array}$ \\
\hline $\begin{array}{l}\text { Quadriceps Muscle Force Output } \\
\qquad \text { (N-m/Kg) } \\
\text { Effect size }\end{array}$ & $\begin{array}{c}0.07 \pm 0.53 \\
(0.17)\end{array}$ & $\begin{array}{l}0.07 \pm 0.36 \\
(0.21)\end{array}$ \\
\hline $\begin{array}{l}\text { Hamstrings Muscle Force Output } \\
\qquad(\mathrm{N}-\mathrm{m} / \mathrm{Kg})\end{array}$ & $\begin{array}{l}0.02 \pm 0.35 \\
\quad(0.08)\end{array}$ & $\begin{array}{c}0.15 \pm 0.34 \\
(0.65)\end{array}$ \\
\hline Y-balance test & $\begin{array}{c}0.02 \pm 0.04 \\
(0.85)\end{array}$ & $\begin{array}{c}0.03 \pm 0.05 \\
(0.66)\end{array}$ \\
\hline
\end{tabular}

Data are reported as raw difference in values (mean \pm SD) with effect size for comparison between the FB and EB intervention. Hamstring flexibility was measured using the popliteus test, which measures popliteal angle (degree) between the thigh and leg. Quadriceps flexibility was measured using Ely's test, which measures the knee flexion angle (degree) between the thigh and leg.

\subsection{Primary Outcomes}

For the hamstring flexibility, the time factor $(p<0.001)$, condition factor $(p=0.04)$, and time factor $\times$ condition factor $(p<0.001)$ were significant. In the post hoc test, participants demonstrated significant improvement after FB intervention relative to their baseline data (immediately, percentage change: $+5.62 \%, p<0.001$, ES $=0.8 ; 20$ min later, percentage change: $+4.95 \%, p<0.001$, ES $=0.72$ ), but did not exhibit significant improvement after EB use (immediately, $p=0.079 ; 20$ min later, $p=0.689$; Table 1 ). Furthermore, compared with the EB intervention results, the FB results indicated significant improvement both immediately $(p<0.001)$ and $20 \mathrm{~min}$ later $(p<0.001)$. The difference between the conditions was associated with a large effect size in favor of FB intervention immediately $(E S=2.09)$ and $20 \mathrm{~min}$ later $(\mathrm{ES}=1.58$; Table 2). For quadriceps flexibility, the time factor $(p=0.642)$ was not significant, but the condition factor $(p=0.006)$ and time factor $\times$ condition factor $(p=0.038)$ were significant. In the post hoc test, participants demonstrated significant improvement after the FB intervention relative to their baseline data (immediately: percentage change $+2.24 \%$, $p<0.001$, ES $=0.32$; 20 min later: percentage change $+2.23 \%, p<0.001$, ES $=0.33$ ), but they did not demonstrate significant improvement after EB intervention (immediately: $p=1$; 20 min later: $p=1$; Table 1). Furthermore, compared with EB intervention, FB intervention resulted in significant improvement both immediately $(p=0.01)$ and 20 min later $(p=0.03)$. The difference between conditions was associated with a medium-to-large effect size in favor of FB intervention immediately $(E S=0.76)$ and 20 min later $(E S=0.84)$; Table 2).

\subsection{Secondary Outcomes}

Regarding knee proprioception, the time factor $(p=0.350)$, condition factor $(p=0.472)$, and time factor $\times$ condition factor $(p=0.551)$ were not significant (Table 1). Furthermore, compared with $\mathrm{EB}$ intervention, $\mathrm{FB}$ intervention resulted in no substantial difference either immediately $(p=0.53)$ and 20 min later $(p=0.4)$.

Regarding quadriceps muscle force output, the time factor $(p<0.001)$ exhibited a significant difference, but the condition factor $(p=0.731)$ and the time factor $\times$ condition factor $(p=0.551)$ were not significant. In the post hoc test, participants demonstrated significant improvement after FB intervention (immediately: $p=0.007, \mathrm{ES}=1.94 ; 20 \mathrm{~min}$ 
later: $p<0.001, \mathrm{ES}=1.8$ ) and $\mathrm{EB}$ intervention (immediately: $p=0.007, \mathrm{ES}=0.36 ; 20 \mathrm{~min}$ later: $p<0.001, \mathrm{ES}=0.25$ ) relative to their baseline data (Table 1 ).

Regarding hamstring muscle force output, the time factor $(p=0.204)$ did not exhibit a significant difference, but the condition factor $(p<0.001)$ and time factor $\times$ condition factor $(p=0.033)$ were significant. In the post hoc test, participants exhibited a significant decrease after FB intervention immediately $(p<0.001, \mathrm{ES}=1.75)$ but not after $20 \mathrm{~min}(p=1$, $\mathrm{ES}=0$ ). However, significant decreases were indicated after EB intervention (immediately: $p<0.001, \mathrm{ES}=1.83 ; 20$ min later: $p<0.001$, ES = 0.32; Table 1). Furthermore, the difference between conditions was associated with a medium-effect size in favor of the FB intervention 20 min later $(\mathrm{ES}=0.65$; Table 2).

Regarding the YBT, the condition factor $(p=0.078)$ was nonsignificant. However, the time factor $(p<0.001)$ and time factor $\times$ condition factor $(p<0.001)$ were significant. In the post hoc test, participants exhibited significant improvement after FB intervention (immediately: $p<0.001 ; 20$ min later: $p<0.001$ ) but not after EB intervention (immediately: $p=0.25$; 20 min later: $p=0.208$; Table 1). Furthermore, compared with EB intervention, FB intervention resulted in significant improvement both immediately $(p=0.016)$ and 20 min later $(p=0.004)$. The difference between conditions was associated with a medium-to-large effect size in favor of FB intervention immediately $(E S=0.85)$ and 20 min later $(E S=0.66$; Table 2).

\section{Discussion}

This is the first study to investigate the effects of FB intervention on the flexibility of the thigh, knee proprioception, muscle force output, and dynamic balance. Our study indicated that participants had significantly improved flexibility of the quadriceps and hamstrings, with a large effect size, immediately following FB intervention, and the effects lasted for $20 \mathrm{~min}$. In addition, participants demonstrated significantly improved quadriceps muscle force output and YBT results. However, knee joint reposition error was not impeded.

The flexibility of the quadriceps and hamstrings was the primary outcome of our study. After wrapping the FB and undergoing 3 min of functional exercises, participants' flexibility was significantly improved. Notably, the effect lasted for at least 20 min after removing the FB. A possible mechanism for an increase in flexibility of quadriceps and hamstrings following a single session of flossing may increase stretch tolerance [5,12] and the thixotropic effect [12]. The use of FB generates compressive pressure on the target muscle, skin, and fascia. This compressive pressure could affect fluid viscosity and lead to less resistance to movement [35]. Regarding the application to the shoulder joint, Kiefer et al. demonstrated that after five 30-second child's pose stretches, the shoulder ROM of the FB-wrapped shoulder improved significantly [2]. By contrast, Plocker et al. indicated that the FB did not achieve a significant difference in shoulder ROM in male athletes; moreover, no improvement in the explosive performance of the upper limbs on the bench press was observed [36]. Regarding the application to the elbow joint, Hodeaux's study demonstrated that using an FB on elite tennis players for 2 min did not significantly improve elbow ROM between the preintervention and postintervention assessments or compared with a bandless control group [37]. In contrast to our study, related studies on elite athletes have observed no significant difference in $\operatorname{ROM}[37,38]$.

Maximum quadriceps force output significantly improved after the 3-min FB intervention. This improvement is supported by a study that indicated that the short-term increase in muscle force output occurred due to the improvement of muscle elasticity after tissue flossing [29]. This finding is also consistent with that of Konrad, who indicated that the maximum voluntary contraction (MVC) knee extensor exhibited increased significant improvement after FB intervention for 2 min on the thighs in healthy men [39]. Possible reasons for an increase in muscle force output following FB intervention could be (1) enhanced growth hormone response, increased sympathetic hormone levels, and improvement in the short latency stretch reflex after the release of the flossing band [7,40,41]; or (2) termed as post-activation potentiation (PAP) and could be provoked after sub maxi- 
mum muscular contraction. The effect may be induced by the enhanced phosphorylation of myosin, increased calcium sensibility, and increased motor neuron excitability [42,43]. However, the maximum strength of the hamstrings decreased significantly in our study. We postulated that the current result may be attributed to the participants being in a sitting position for the Biodex exercise rather than the desired prone position; if the isokinetic maximum muscle strength is measured in this position, the hamstring muscles may be placed in a relatively unfavorable position for contraction because the muscles are being compressed. As is consistent with Vorgin's findings, MVC knee flexors exhibited no significant change in recreationally trained adults [4]. Additionally, in Driller's and Mills' studies on professional rugby players, athletic performance (i.e., sprinting and jumping) after FB intervention increased slightly immediately after banding, but the increase was not significant, and its status returned to the preintervention level after $15 \mathrm{~min}[15,38]$. Therefore, further investigation of the magnitude of change of muscle force output is required.

Less change in the knee joint reposition angle error and sound dynamic balance is imperative to reduce risk factors for sports injury in the lower limbs. In this study, we provided the first data verifying that FB intervention on the knee joint may not result in proprioception changes at all time points; thus, the knee joint is not impeded. Proprioception provides expedient feedback on joint stability; as long as joint proprioception is provoked, it is a crucial factor that can increase susceptibility to knee sprain joint injury. The proprioceptive performance depends on the ability of the central nervous system to use other sensory inputs (e.g., the muscles, ligaments, tendons, joint capsules, and skin), theoretically, which could be trained through long-term learning and neuroplasticity mechanisms [44,45]. Nevertheless, nuanced interpretation of this data is paramount. We employed open kinetic chain $(\mathrm{OKC})$, not closed kinetic chain $(\mathrm{CKC})$, measurement in participants. Further comparison between OKC and CKC measurements is thus suggested. Regarding dynamic balance, our study demonstrated that FB intervention resulted in significant improvement with a medium-to-large effect in the YBT assessment in immediate postintervention (percentage change: $4.43, p<0.001$, ES $=0.57$ ) and $20 \mathrm{~min}$ postintervention (percentage change: $5.77, p<0.001, \mathrm{ES}=0.86$ ). Two potential reasons were postulated: (1) soft tissues were relaxed and muscle elasticity increased, thereby reducing joint tightness and increasing the performance of dynamic balance; consistent with the literature, this outcome indicates that the increased ROM is positively correlated with dynamic balance determined by the YBT [46]; and (2) the improvements in dynamic balance and isokinetic quadriceps strength are related [24]. Because the quadriceps muscle acts as a dynamic stabilizer of the knee joint, it aids in dynamic balance. We also determined that the participants exhibited significant improvement in quadriceps muscle force output after FB intervention both immediately $(\mathrm{ES}=1.94)$ and $20 \mathrm{~min}$ later $(\mathrm{ES}=1.8)$.

This study has some limitations. First, no consensus exists on the optimal FB use technique $[12,17]$. Second, the pressure on the target tissue after flossing was not measured since an athletic trainer or physiotherapist cannot wrap the FB with exactly the same pressure on the court. Thus, we used the Smart Handle Pro ZSH-ZP to control the tension of the stretched FB for the purpose of a more practical approach. Hodeaux's research demonstrated that different stretched tensions of the FB resulted in distinct outcomes [37], and the band was stretched to a point where the estimated force had already been assessed; therefore, the average band tension was fixed at $40 \mathrm{~N}$ in this study [29]. However, we did not further investigate whether lower or higher band stretching, varying force, or pressure on the target tissue may lead to varying results $[4,47]$. Third, the percentage of blood restriction was not assessed by a laser Doppler flowmeter. Different applied blood restrictions of the body segment on the target muscle during FB intervention may have generated varied outcomes. Fourth, this study was performed with only healthy individuals. The generalizability of the results to all populations (e.g., participants with musculoskeletal disorders, recreational, or elite athletes) is relatively low. Fifth, muscle activity (e.g., electromyography) was not measured. Sixth, further investigation may also focus on band use in different joints and soft tissues. Lastly, our study compared FB and 
EB (ie., sham control condition), but the same testing protocol could have been repeated without any wrapping as the control condition (i.e., untreated condition). In addition, the compression materials are different. The EB is a made of a mix of cotton and elastic yarn [19]. FB is a made of latex rubber. Therefore, the wrapping time and stretched tension are comparable, but the different bands have different structures and degrees of elasticity, which may affect the BFR of the targeted tissues and corresponding physiological effects.

For practical application, the results of this investigation indicate that FB intervention can significantly improve the flexibility of the quadriceps and hamstrings, quadriceps muscle force output, and dynamic balance without impeding knee proprioception. Using this technique in a warm-up program is a feasible because the time of athletic practice is very limited. We speculate that recreational active men without musculoskeletal disorders may adopt an FB program under athletic professionals' assistance. This program simply includes three functional movements (walking knee lift, side squat, and lunge) and 10 repetitions in $3 \mathrm{~min}$ at 30 beats per min. Athletes, coaches, athletic professionals and/or physiotherapists may consider them when selecting FB practices as a potential warmup to augment flexibility and quadriceps muscle force output, and dynamic balance in young adults. The findings may soon be useful in athletic practice settings. However, it should be noted that contraindications of the FB include latex allergy, deep vein thrombosis, varicose veins, open wounds, fractures, skin symptoms, obstructive vascular diseases, arterial occlusive diseases, acute arthritis, malignant tumor disease, and nerve or known cardiovascular and metabolic diseases $[13,18]$.

\section{Conclusions}

Through a single session of FB use, participants demonstrated significantly improved flexibility of the quadriceps and hamstrings, with a large effect size immediately following FB intervention, and the effects lasted for $20 \mathrm{~min}$. In addition, participants demonstrated significantly improved quadriceps muscle force output and YBT results. However, knee proprioception (i.e., joint reposition angle error) was not impeded.

Author Contributions: Conceptualization, N.-J.C., W.-C.H. and C.-L.L.; Methodology, N.-J.C., W.-C.H., C.-L.L., W.-D.C. and B.-H.W.; Investigation, N.-J.C. and W.-C.H.; Resources, N.-J.C., W.-C.H. and C.-L.L.; Data Curation N.-J.C., W.-C.H. and C.-L.L.; Writing-Original Draft Preparation, N.-J.C. and W.-C.H.; Writing-Review and Editing, N.-J.C., W.-C.H., C.-L.L., W.-D.C. and B.-H.W.; Supervision, N.-J.C.; Project Administration, N.-J.C. and C.-L.L. All authors have read and agreed to the published version of the manuscript.

Funding: This research was supported by the NSYSU-KMU joint research projects [NSYSUKMU108P037; 110-P004]. This work was also in part supported by the NPUST-KMU joint research project [NPUST-KMU-109-P011] and by the Ministry of Science and Technology of Taiwan (MOST 110-2410$\mathrm{H}-037-014)$.

Institutional Review Board Statement: The study was conducted according to the guidelines of the Declaration of Helsinki and approved by the Kaohsiung Medical University Hospital Institutional Review Board (KMU-HIRB-F(I)-20190063).

Informed Consent Statement: Informed consent was obtained from all subjects involved in the study.

Data Availability Statement: Data are contained within the article.

Acknowledgments: We would like to thank all our participants for their time and effort.

Conflicts of Interest: The authors declare no conflict of interest.

\section{References}

1. Opplert, J.; Babault, N. Acute Effects of Dynamic Stretching on Muscle Flexibility and Performance: An Analysis of the Current Literature. Sports Med. 2018, 48, 299-325. [CrossRef] [PubMed]

2. Kiefer, B.; Lemarr, K.; Enriquez, C.; Tivener, K. A Pilot Study: Psychological Effects of the Voodoo Floss Band on Glenohumeral Flexibility. Int. J. Athl. Ther. Train. 2017, 22, 1-16. 
3. Starrett, K.; Cordoza, G. Becoming a Supple Leopard: The Ultimate Guide to Resolving Pain, Preventing Injury, and Optimizing Athletic Performance, 2nd ed.; Victory Belt Publishing: Las Vegas, NV, USA, 2013.

4. Vogrin, M.; Kalc, M.; Licen, T. Acute Effects of Tissue Flossing around the Upper Thigh on Neuromuscular Performance: A Study Using Different Degrees of Wrapping Pressure. J. Sport Rehabil. 2020, 30, 601-608. [CrossRef]

5. Kaneda, H.; Takahira, N.; Tsuda, K.; Tozaki, K.; Kudo, S.; Takahashi, Y.; Sasaki, S.; Kenmoku, T. Effects of Tissue Flossing and Dynamic Stretching on Hamstring Muscles Function. J. Sports Sci. Med. 2020, 19, 681-689. [PubMed]

6. Reeves, G.V.; Kraemer, R.R.; Hollander, D.B.; Clavier, J.; Thomas, C.; Francois, M.; Castracane, V.D. Comparison of hormone responses following light resistance exercise with partial vascular occlusion and moderately difficult resistance exercise without occlusion. J. Appl. Physiol. 2006, 101, 1616-1622. [CrossRef] [PubMed]

7. Takarada, Y.; Nakamura, Y.; Aruga, S.; Onda, T.; Miyazaki, S.; Ishii, N. Rapid increase in plasma growth hormone after low-intensity resistance exercise with vascular occlusion. J. Appl. Physiol. 2000, 88, 61-65. [CrossRef]

8. Loenneke, J.P.; Wilson, J.M.; Marín, P.J.; Zourdos, M.C.; Bemben, M.G. Low intensity blood flow restriction training: A metaanalysis. Eur. J. Appl. Physiol. 2012, 112, 1849-1859. [CrossRef]

9. Pope, Z.K.; Willardson, J.M.; Schoenfeld, B.J. Exercise and blood flow restriction. J. Strength Cond. Res. 2013, 27, 2914-2926. [CrossRef]

10. Lawson, C.S.; Downey, J.M. Preconditioning: State of the art myocardial protection. Cardiovasc. Res. 1993, 27, 542-550. [CrossRef]

11. Pang, C.Y.; Yang, R.Z.; Zhong, A.; Xu, N.; Boyd, B.; Forrest, C.R. Acute ischaemic preconditioning protects against skeletal muscle infarction in the pig. Cardiovasc. Res. 1995, 29, 782-788. [CrossRef]

12. Konrad, A.; Mocnik, R.; Nakamura, M. Effects of Tissue Flossing on the Healthy and Impaired Musculoskeletal System: A Scoping Review. Front. Physiol. 2021, 12, 666129. [CrossRef]

13. Pavlů, D.; Pánek, D.; Kuncová, E.; Thung, J.S. Effect of Blood Circulation in the Upper Limb after Flossing Strategy. Appl. Sci. 2021, 11, 1634. [CrossRef]

14. Wortman, R.J.; Brown, S.M.; Savage-Elliott, I.; Finley, Z.J.; Mulcahey, M.K. Blood Flow Restriction Training for Athletes: A Systematic Review. Am. J. Sports Med. 2021, 49, 1938-1944. [CrossRef] [PubMed]

15. Driller, M.W.; Overmayer, R.G. The effects of tissue flossing on ankle range of motion and jump performance. Phys. Ther. Sport 2017, 25, 20-24. [CrossRef] [PubMed]

16. Vogrin, M.; Novak, F.; Licen, T.; Greiner, N.; Mikl, S.; Kalc, M. Acute Effects of Tissue Flossing on Ankle Range of Motion and Tensiomyography Parameters. J. Sport Rehabil. 2020, 30, 129-135. [CrossRef] [PubMed]

17. Kielur, D.S.; Powden, C.J. Changes of Ankle Dorsiflexion Using Compression Tissue Flossing: A Systematic Review and Meta-Analysis. J. Sport Rehabil. 2020, 30, 306-314. [CrossRef] [PubMed]

18. Kruse, S. Easy Flossing, 1st ed.; Georg Thieme Verlag KG: Stuttgart, Germany, 2018.

19. Kumar, B.; Das, A.; Alagirusamy, R. Effect of material and structure of compression bandage on interface pressure variation over time. Phlebology 2014, 29, 376-385. [CrossRef] [PubMed]

20. Ahlhorn, A.; Krämer, D. Flossing in Therapie und Training; Riva: Munich, Germany, 2016.

21. Suresh, K. An overview of randomization techniques: An unbiased assessment of outcome in clinical research. J. Hum. Reprod. Sci. 2011, 4, 8-11. [CrossRef] [PubMed]

22. Youdas, J.W.; Krause, D.A.; Hollman, J.H.; Harmsen, W.S.; Laskowski, E. The influence of gender and age on hamstring muscle length in healthy adults. J. Orthop. Sports Phys. Ther. 2005, 35, 246-252. [CrossRef] [PubMed]

23. Butler, R.J.; Southers, C.; Gorman, P.P.; Kiesel, K.B.; Plisky, P.J. Differences in soccer players' dynamic balance across levels of competition. J. Athl. Train. 2012, 47, 616-620. [CrossRef]

24. Lee, C.L.; Chu, I.H.; Lyu, B.J.; Chang, W.D.; Chang, N.J. Comparison of vibration rolling, nonvibration rolling, and static stretching as a warm-up exercise on flexibility, joint proprioception, muscle strength, and balance in young adults. J. Sports Sci. 2018, 36, 2575-2582. [CrossRef]

25. Callaghan, M.J.; Selfe, J.; Bagley, P.J.; Oldham, J.A. The Effects of Patellar Taping on Knee Joint Proprioception. J. Athl. Train. 2002, 37, 19-24. [CrossRef]

26. Drouin, J.M.; Valovich-mcLeod, T.C.; Shultz, S.J.; Gansneder, B.M.; Perrin, D.H. Reliability and validity of the Biodex system 3 pro isokinetic dynamometer velocity, torque and position measurements. Eur. J. Appl. Physiol. 2004, 91, 22-29. [PubMed]

27. Charlier, R.; Mertens, E.; Lefevre, J.; Thomis, M. Muscle mass and muscle function over the adult life span: A cross-sectional study in Flemish adults. Arch. Gerontol. Geriatr. 2015, 61, 161-167. [CrossRef]

28. Linek, P.; Sikora, D.; Wolny, T.; Saulicz, E. Reliability and number of trials of Y Balance Test in adolescent athletes. Musculoskelet. Sci. Pract. 2017, 31, 72-75. [CrossRef] [PubMed]

29. Cheatham, S.W.; Baker, R. Technical Report: Quantification of the Rockfloss ${ }^{\circledR}$ Floss Band Stretch Force at Different Elongation Lengths. J. Sport Rehabil. 2019, 29, 377-380. [CrossRef] [PubMed]

30. Haff, G.G.; Triplett, N.T. Essentials of Strength Training and Conditioning, 4th ed.; Human Kinetics: Champaign, IL, USA, 2015.

31. Faul, F.; Erdfelder, E.; Lang, A.G.; Buchner, A. G*Power 3: A flexible statistical power analysis program for the social, behavioral, and biomedical sciences. Behav. Res. Methods 2007, 39, 175-191. [CrossRef]

32. Lyu, B.J.; Lee, C.L.; Chang, W.D.; Chang, N.J. Effects of Vibration Rolling with and without Dynamic Muscle Contraction on Ankle Range of Motion, Proprioception, Muscle Strength and Agility in Young Adults: A Crossover Study. Int. J. Environ. Res. Public Health 2020, 17, 354. [CrossRef] [PubMed] 
33. Lee, S.; Lee, D.K. What is the proper way to apply the multiple comparison test? Korean J. Anesthesiol. 2020, 73, 572. [CrossRef]

34. Lakens, D. Calculating and reporting effect sizes to facilitate cumulative science: A practical primer for $t$-tests and ANOVAs. Front. Psychol. 2013, 4, 863. [CrossRef]

35. Behm, D.G.; Wilke, J. Do Self-Myofascial Release Devices Release Myofascia? Rolling Mechanisms: A Narrative Review. Sports Med. 2019, 49, 1173-1181. [CrossRef] [PubMed]

36. Plocker, D.; Wahlquist, B.; Dittrich, B. Effects of tissue flossing on upper extremity range of motion and power. Int. J. Exerc. Sci. Conf. Proc. 2015, 12, 37.

37. Hodeaux, K. The Effect of Floss Bands on Elbow Range of Motion in Tennis Players. Bachelor's Thesis, University of Arkansas, Fayetteville, NC, USA, 2017.

38. Mills, B.; Mayo, B.; Tavares, F.; Driller, M. The Effect of Tissue Flossing on Ankle Range of Motion, Jump, and Sprint Performance in Elite Rugby Union Athletes. J. Sport Rehabil. 2019, 29, 282-286. [CrossRef]

39. Konrad, A.; Bernsteiner, D.; Budini, F.; Reiner, M.M.; Glashuttner, C.; Berger, C.; Tilp, M. Tissue flossing of the thigh increases isometric strength acutely but has no effects on flexibility or jump height. Eur. J. Sport Sci. 2020, 21, 1648-1658. [CrossRef]

40. Driller, M.; Mackay, K.; Mills, B.; Tavares, F. Tissue flossing on ankle range of motion, jump and sprint performance: A follow-up study. Phys. Ther. Sport 2017, 28, 29-33. [CrossRef] [PubMed]

41. Hjortskov, N.; Skotte, J.; Hye-Knudsen, C.; Fallentin, N. Sympathetic outflow enhances the stretch reflex response in the relaxed soleus muscle in humans. J. Appl. Physiol. 2005, 98, 1366-1370. [CrossRef] [PubMed]

42. Hodgson, M.; Docherty, D.; Robbins, D. Post-activation potentiation: Underlying physiology and implications for motor performance. Sports Med. 2005, 35, 585-595. [CrossRef]

43. Mitchell, C.J.; Sale, D.G. Enhancement of jump performance after a 5-RM squat is associated with postactivation potentiation. Eur. J. Appl. Physiol. 2011, 111, 1957-1963. [CrossRef]

44. Ager, A.L.; Borms, D.; Deschepper, L.; Dhooghe, R.; Dijkhuis, J.; Roy, J.S.; Cools, A. Proprioception: How is it affected by shoulder pain? A systematic review. J. Hand Ther. 2020, 33, 507-516. [CrossRef]

45. Roijezon, U.; Clark, N.C.; Treleaven, J. Proprioception in musculoskeletal rehabilitation. Part 1: Basic science and principles of assessment and clinical interventions. Man. Ther. 2015, 20, 368-377. [CrossRef]

46. Aslan, H.; Buddhadev, H.H.; Suprak, D.N.; San Juan, J.G. Acute effects of two hip flexor stretching techniques on knee joint position sense and balance. Int. J. Sports Phys. Ther. 2018, 13, 846-859. [CrossRef] [PubMed]

47. Galis, J.; Cooper, D.J. Application of a Floss Band at Differing Pressure Levels: Effects at the Ankle Joint. J. Strength Cond. Res. 2020. [CrossRef] 